\title{
RELACIÓN ENTRE EL GRADO DE INCONTINENCIA FECAL Y EL DAÑO DEL ESFÍNTER ANAL EXTERNO*
}

\author{
Drs. Antonella Sanguineti M. ${ }^{1}$, Andrés Larach K. ${ }^{2}$, Katya Carrillo G. ${ }^{1}$, \\ Alejandro Zárate C. ${ }^{4}$, Francisco López K. ${ }^{2}$, Claudio Wainstein G. ${ }^{2}$, \\ Udo Kronberg ${ }^{2}$, Jorge Larach S. ${ }^{2}$, Patricio Burdiles P. ${ }^{3}$
}

1 Alumna. Magíster en Ciencias Médicas y Biológicas, con mención en Morfología. Universidad de Chile.

2 Unidad de Coloproctología y Departamento de Cirugía, Clínica Las Condes.

3 Departamento de Cirugía, Clínica Las Condes.

4 Servicio de Urgencia, Clínica Las Condes.

Santiago, Chile.

\section{Abstract \\ Relationship between faecal incontinence severity and external anal sphincter disruption}

Introduction: Faecal incontinence (FI) represents an important psychological and social condition for an individual. There are several causes for FI, therefore its study and management is complex. Among different aetiologies, anatomical disruption of the external anal sphincter (EAS) as a consequence of anorrectal surgery or obstetric injury can be studied by endoanal ultrasound (EAUS). This study focus on anatomical assessment of sphincter muscle injury of EAS and its relationship with clinical presentation con FI. Objective: Compare anatomical and disruption of EAS features studied by 3D-EAUS between patients with FI, according to its severity. Methods: 3D-EAUS images were obtained from selected patients studied for FI between January 2008 and July 2010 at Clínica las Condes. Wexner Score (SW) was used to evaluate severity of FI, dividing patients into two categories: Mild FI $(\mathrm{SW}<9)$ and Severe FI $(\mathrm{SW} \geq 9)$. A single observer evaluated different morphological variables: width, length, percentage and angle of EAS disruption. Parametrical and non-parametrical analysis was used as appropriate. P-value $<0.05$ was considered statistically significant. Results: From 31 female patients studied, mean SW was $11.7 \pm 0.8$ points, range from 5 to 20 points. When dividing into two groups, 9 patients had mild FI and 22 had severe FI. Studied variables in EAUS 3D cubes: width, length, percentage and angle of EAS disruption, did not reach statistical significance between groups. Conclusion: No relationship was found between external anal sphincter anatomic injury variables studied and severity of faecal incontinence.

Key words: Faecal incontinence, external anal sphincter, endoanal ultrasound.

\footnotetext{
Los autores no refieren conflictos de interés.

Correspondencia: Dra. Antonella Sanguineti M. asanguineti@gmail.com
}

*Recibido el 2 de enero de 2015 y aceptado para publicación el 31 de enero de 2015. 


\section{Resumen}

Introducción: La incontinencia fecal (IF) se refleja en una condición psicológica y social importante para el individuo. Las causas de IF son múltiples, siendo su estudio y manejo complejo. La disrupción anatómica del esfínter anal externo (EAE), secundaria, entre otras, a cirugía anorrectal o lesión obstétrica, es posible de ser estudiada por endosonografía anal (EAUS). El presente artículo se centra en el daño anatómico del EAE y su relación con la presentación clínica de la IF. Objetivo: Comparar las características anatómicas y daño del EAE objetivadas mediante endosonografía en 3 dimensiones (EAUS3D), entre los pacientes con IF según su severidad. Material y Método: Se obtuvo las EAUS3D realizadas en Clínica Las Condes por estudio de IF entre enero de 2008 y julio de 2010. Se utilizó el score de Wexner (SW) para separar la población en dos grupos, IF leve $(\mathrm{SW}<9)$ e IF grave $(\mathrm{SW} \geq 9)$. Un único observador evaluó las diferentes variables: grosor, longitud, porcentaje de defecto y ángulo de lesión del EAE. Se utilizó estadística paramétrica o no-paramétrica según corresponda. Se consideró significativo $p<0,05$. Resultados: De un total de 31 pacientes femeninas estudiadas, el SW promedio fue de $11,7 \pm 0,8$ con rango entre 5 y 20 puntos. Al separar según grupos, 9 pacientes tenían IF leve y 22 IF grave. De las variables estudiadas por EAUS3D: el promedio, grosor, longitud, porcentaje de defecto y el ángulo de lesión del EAE no lograron diferencia estadística entre los grupos. Conclusión: No existe una relación directa entre las variables estudiadas de daño anatómico del EAE y el grado de IF.

Palabras clave: Incontinencia Fecal, esfínter anal externo, endosonografía anal.

\section{Introducción}

La incontinencia fecal (IF) representa una condición psicológica y social importante y muy debilitante para el individuo.

Las causas de la incontinencia fecal (IF) son múltiples.

Para lograr una continencia efectiva participan numerosos factores, entre los cuales destacan: estructuras anatómicas sin alteraciones, capacidad de dilatación rectal para el almacenamiento fecal, contracción voluntaria del piso pélvico y del esfínter anal externo (EAE) y tono muscular del esfínter anal interno (EAI). Además de la indemnidad del sistema nervioso, consistente en: la conservación de los automatismos y de los reflejos medulares y del tronco cerebral, de la propiocepción en los músculos estriados del suelo pélvico y la sensibilidad en la mucosa del conducto anal. A lo anterior se agrega la consistencia de las heces ${ }^{1,2}$.

Una sola falla de estos factores no explicaría la alteración en la continencia normal, si a este se agrega un segundo o tercer factor suele originar incontinencia fecal ${ }^{3}$.

Existen múltiples escalas para evaluar la gravedad de la IF y la calidad de vida (CV) de estos pacientes. Actualmente la más usada es la escala de Jorge-Wexner o CCFIS (Tabla 1), útil para poder objetivar el resultado de los diferentes tratamientos. Esta evalúa la frecuencia de los episodios de incontinencia, el tipo de deposiciones, incontinencia a gases y la necesidad de uso de protectores o toallas higiénicas. Además incluye el compromiso en la calidad de vida.

Rothbarth, en el año 2007 mediante un estudio ${ }^{4}$, determinó cual es el punto de la IF medida por el score de Wexner o CCF-FIS que afecta significativamente la calidad de vida del individuo. Como resultado se obtuvo que una puntuación en el score de Wexner de 9 o más, indica un deterioro significativo de la CV y por lo tanto, se puede utilizar en la toma de decisiones del tratamiento. La puntuación de Wexner se puede hacer fácilmente y a diferencia de las escalas de $\mathrm{CV}$, no está influenciado por enfermedades concomitantes ${ }^{3}$.

En este trabajo nos centraremos en la lesión muscular esfinteriana como causa de la incontinencia fecal, susceptible de estudiar con endosonografía anal (EAUS). Esta tiene una sensibilidad y especificidad para la detección de defectos esfinterianos entre un 83 y $100 \%$ en la mayoría de estudios 5 .

El objetivo general es determinar, mediante estudios endosonográficos anales (EAUS) en 3 dimen-

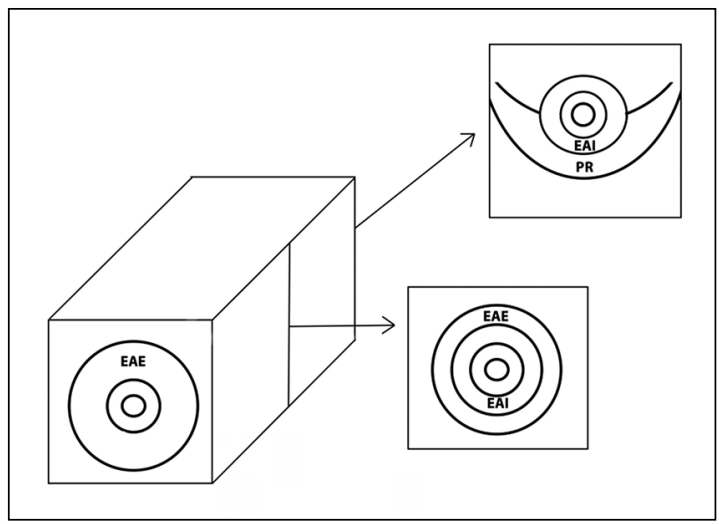

Figura 1. Esquema del conducto anal visto por EAUS3D. 
Tabla 1. Score de Wexner (español)

\begin{tabular}{|lccccc|}
\hline Tipo IF & Nunca & $\begin{array}{c}\text { Raramente } \\
(<\mathbf{1} \text { vez/mes })\end{array}$ & $\begin{array}{c}\text { Algunas veces } \\
(>\mathbf{1} \text { vez/mes y } \\
<\mathbf{1} \text { vez/semana) }\end{array}$ & $\begin{array}{c}\text { Frecuente } \\
(>\mathbf{1} \text { vez/semana } \\
\mathbf{y}<\mathbf{1} \text { vez/día) }\end{array}$ & $\begin{array}{c}\text { Siempre } \\
(>\mathbf{1} \text { vez/día) }\end{array}$ \\
Gases & 0 & 1 & 2 & 3 & 4 \\
Líquidas & 0 & 1 & 2 & 3 & 4 \\
Sólidas & 0 & 1 & 2 & 3 & 4 \\
Uso de Paños & 0 & 1 & 2 & 3 & 4 \\
Alteración de estilo de vida & 0 & 1 & 2 & 3 & 4 \\
\hline
\end{tabular}

0 : Continencia perfecta. 20: Incontinencia completa.

siones, el daño del EAE que muestran los pacientes con IF, separados por una puntuación de score de Wexner igual o mayor a 9.

Los objetivos específicos incluyen determinar la IF leve y la IF grave mediante la puntuación del score de Wexner, determinar las variables a estudiar en la EAUS ${ }^{6} \mathrm{y}$, por último, evaluar la relación de las variables estudiadas en la endosonografía anal, con la IF leve y la IF grave.

\section{Material y Método}

Se recopilaron de forma consecutiva los exámenes EUAS3D realizados en Clínica Las Condes en período comprendido entre enero del año 2008 y julio de 2010, solicitados por el diagnóstico de IF. No se incluyó a los pacientes de los que no se disponía de antecedentes por ser atendidos en otro centro.

Se obtuvo datos demográficos y Score de Wexner de cada paciente de ficha clínica médica o kinesiológica. Se clasificó a los pacientes en dos grupos: el primero o IF leve, pacientes con un Score de Wexner menor a 9 y el segundo grupo o IF grave, con una puntuación igual o mayor a 9 .

La EAUS3D se realizó mediante el Ecógrafo BK, ProFocus modelo 2202 con un transductor 3D BK 2050 , de 6-16MHz. Se extrajo los cubos tridimensionales de información de la EAUS3D (Figura 1) los que fueron evaluados por un único observador entrenado. Se midió las variables: 1) Largo total del conducto anal, medido desde el haz puborrectal hasta el límite inferior del esfínter anal interno (EAI); 2) Largo total del defecto del esfínter anal externo (EAE), medido desde el haz puborrectal hasta el punto en que se reconoce nuevamente EAE indemne; 3) Grosor del EAE, a nivel del conducto anal medio; 4) Ángulo del defecto, a nivel del conducto anal medio (Figura 2); 5) Porcentaje del defecto del EAE, calculado por la proporción entre el largo del defecto del EAE y el largo total del conducto anal (Figura 3).

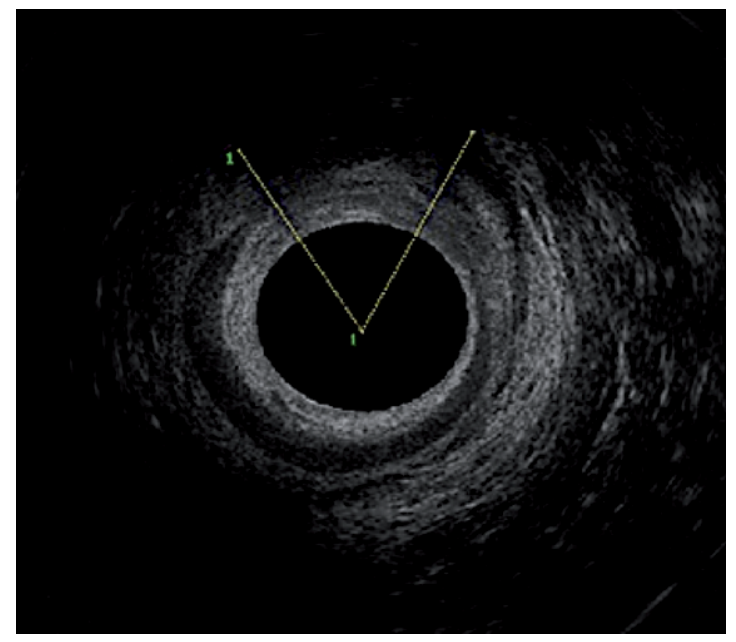

Figura 2. Ángulo del defecto, a nivel del conducto anal medio.

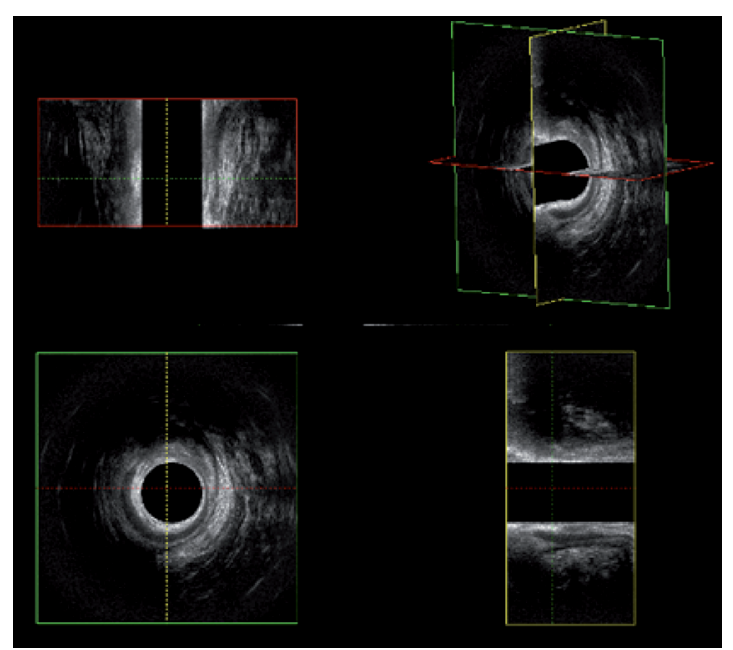

Figura 3. Diferentes cortes de EAUS3D que permiten obtener porcentaje de daño del EAE. 
Para el análisis de ambos grupos (IF leve e IF grave) se realizó un análisis de distribución para cada variable mediante el test de Shapiro-Willk. Según la distribución paramétrica o no paramétrica de ésta se aplicó los test estadísticos correspondientes para el análisis de dos muestras independientes. El análisis fue realizado en el programa estadístico SPSS versión 20. Se consideró un p value $<0,05$ como significativo.

\section{Resultados}

De las 45 EUS3D realizadas en este período, se reclutó un total de 31 pacientes, excluyendo a aquellos sin datos o con datos incompletos.

Todas eran de sexo femenino, con edad promedio de $60,1 \pm 10,35$ años. Entre los antecedentes obstétricos, sólo una paciente era nulípara y, de las 30 restantes, el 93,3\% habían tenido al menos un parto vaginal, $20 \%$ al menos 4 partos vaginales, con un promedio de 2,3 $\pm 0,3$ partos. El $36,7 \%$ de las multíparas habían tenido partos operatorios con utilización de fórceps.

El Score de Wexner promedio fue de 11,7 $\pm 0,8$ con un rango entre 5 y 20 puntos. Al separar según grupos, 9 pacientes tenían IF leve $(\mathrm{SW}<9)$ y 22 IF grave $(\mathrm{SW} \geq 9)$. Las características demográficas de ambos grupos se resumen en la Tabla 2.

En cuanto a los datos obtenidos de los cubos 3D de EAUS, se obtuvo un grosor y longitud promedio

Tabla 2. Datos demográficos

\begin{tabular}{|lccc|}
\hline Variable & $\begin{array}{c}\text { IF Leve } \\
\text { (SW }<\mathbf{9 )}\end{array}$ & $\begin{array}{c}\text { IF Grave } \\
(\mathbf{S W} \geq \mathbf{9 )}\end{array}$ & $\begin{array}{c}\mathbf{p} \\
\text { value }\end{array}$ \\
\hline Edad (años) & $58 \pm 3$ & $61,5 \pm 2,3$ & 0,36 \\
Partos Total & $3 \pm 0,44$ & $2,73 \pm 0,3$ & 0,62 \\
Partos Vaginales & $2,33 \pm 0,47$ & $2,27 \pm 0,33$ & 0,92 \\
Uso de Fórceps (\%) & $22 \pm 0,15$ & $41 \pm 0,1$ & 0,43 \\
\hline
\end{tabular}

de $3,3 \pm 0,12 \mathrm{~mm}$ y $22,93 \pm 0,6 \mathrm{~mm}$, respectivamente. Los valores promedio de las características endosonográficas de los defectos anatómicos del EAE fueron $83,7 \pm 8,5^{\circ}$ para el ángulo del defecto, $13,3 \pm 1,4 \mathrm{~mm}$ para la longitud del defecto y 57,4 $\pm 5,9 \%$ para el porcentaje del defecto en relación a la longitud total del EAE. Las diferencias entre ambos grupos, IF leve en comparación con IF grave, se resumen en la Tabla 3. Ninguna de las variables medidas logró una diferencia estadísticamente significativa.

\section{Discusión}

La IF presenta una prevalencia con alta variabilidad en la población general ${ }^{7,8}$. Debido al considerable efecto que tiene la IF en la calidad de vida (CV) de los pacientes tanto en el ámbito sicológico como social ${ }^{9,10}$, es importante encontrar los factores que condicionan esta patología. Lo anterior no es fácil de hacer ya que un alto número de pacientes afectados no consulta por el estigma que esta conlleva ${ }^{11}$.

Por tanto, la cantidad de estudios imagenológicos realizados para estudiar la IF, no se relacionan con el real número de afectados. Esto incide en el número de pacientes incluidos en los estudios ${ }^{11}$.

Al realizar una búsqueda en la literatura para poder comparar nuestros resultados, nos encontramos con numerosos trabajos que estudian la IF evaluando las alteraciones del conducto anal mediante diferentes métodos diagnósticos, como la EAUS, la manometría endoanal ${ }^{12}$, la resonancia magnética endoanal ${ }^{13}$ y la ultrasonografía transperineal ${ }^{14}$. Estos trabajos se enfocan en comparar los métodos de estudio y en analizar la presencia de alteraciones en un grupo con IF versus un grupo control.

Lo que nos muestran los diferentes trabajos que estudian la IF con EAUS es que existe una relación entre el daño que presenta el aparato esfinteriano y la IF.

Lo anterior puede estar dado ya sea por una disrupción del EAE o EAI, que ocurre principalmente

Tabla 3. Variables y su comparación en ambos grupos

\begin{tabular}{|lccc|}
\hline Variable & IF Leve $(\mathbf{S W}<\mathbf{9})$ & IF Grave $(\mathbf{S W} \geq \mathbf{9})$ & p value \\
\hline Grosor EAE $(\mathrm{mm})$ & $3,11 \pm 0,2$ & $3,36 \pm 0,16$ & 0,43 \\
\hline Longitud EAE $(\mathrm{mm})$ & $23 \pm 0,8$ & $22,9 \pm 0,77$ & 0,94 \\
\hline Ángulo del Defecto EAE $\left({ }^{\circ}\right)$ & $96,56 \pm 13,31$ & $78,59 \pm 10,25$ & 0,24 \\
Longitud del Defecto EAE $(\mathrm{mm})$ & $15,22 \pm 2,13$ & $12,77 \pm 1,77$ & 0,48 \\
\hline Porcentaje del Defecto EAE $(\%)$ & $67,11 \pm 10,11$ & $54,61 \pm 7,05$ & 0,22 \\
\hline
\end{tabular}


por el daño obstétrico ${ }^{17-20}$ en pacientes jóvenes y sanas en su mayoría asintomáticas hasta la menopausia, así como también por un adelgazamiento esfinteriano observado en pacientes añosos ${ }^{21}$. Kim YS et al. ${ }^{22}$, demostró que esta alteración anatómica de ambos esfínteres, se correlaciona con alteraciones de la contracción en la manometría. Sin embargo, si bien los estudios parecen demostrar la relación entre el daño esfinteriano y la presencia de IF, aún no hay resultados que muestren si este grado de IF se relaciona con el nivel de compromiso del aparato esfinteriano. Es por lo anterior que los resultados de este estudio son interesantes, dado que no se demostró una relación directa entre el grado de IF y la magnitud de la disrupción anatómica de EAE. Esto es importante al momento de evaluar al paciente portador de IF, con el fin de poder elegir el mejor tratamiento. A pesar de que existen múltiples opciones de tratamiento, ya sea conservador como la neuromodulación, el biofeedback, la terapia hormonal sistémica y local ${ }^{23}$, mejorar la actividad física en los pacientes ancianos ${ }^{21}$; así como también el tratamiento quirúrgico, ninguno de ellos ha demostrado tener gran efectividad. Entonces, los resultados de éste y otros estudios llevan a plantear la pregunta de si la opción quirúrgica es la mejor alternativa en este grupo de pacientes ya que la reparación anatómica esfinteriana no asegura una mejoría en la continencia.

\section{Conclusión}

Los resultados de este estudio, realizado con EAUS3D, demuestran que el daño del EAE medido en todas sus variables, no es responsable único directo de IF. Se infiere que el daño del EAE debe estar asociado a otras alteraciones en los mecanismos de continencia, para llevar a una IF grave.

\section{Referencias}

1. Moore K, Dalley A. Anatomía, con orientación clínica. $5^{\mathrm{a}}$ Edición. Hong Kong, China: Editorial Médica Panamerica, 2007;355-435.

2. Carlson B. Embriología humana y biología del desarrollo. $4^{\mathrm{a}}$ Edición. Madrid, España: Elsevier Saunders, 2009;361-9.

3. Jorge JM, Wexner SD. Etiology and management of fecal incontinence. Dis Colon Rectum 1993;36:77-97.

4. Rothbarth J, Bemelman WA, Meijerink WJ, Stiggelbout AM, Zwinderman AH, Buyze-Westerweel ME, et al. What is the impact of fecal incontinence on quality of life? Dis Colon Rectum 2001;44:67-71.

5. Damon H, Henry L, Barth X, Valette PJ, Mion F. Anal incontinence: echographic and manometric study. Ann Chir. 2001;126:869-75.
6. De la Portilla F. Principios prácticos de ecografía anal y rectal. Madrid, España: Ediciones Díaz de Santos, 2004;19-30.

7. Varma M, Brown J, Creasman J, Thom D, Van Den Eeden S, Beattie, M, et al. Fecal Incontinence in Females Older Than Aged 40 Years: Who is at Risk? Dis Colon Rectum 2006;49:841-51.

8. Zárate AJ, López F, Vergara F, Badilla N, Viviani P. Prevalencia de la incontinencia fecal en Centros de Salud y Casas de Reposo. Rev Med Chile 2008;136:867-72.

9. Norton C, Whitehead W. Conservative and pharmacological management of faecal incontinence in adults. In Abrams P, Cardozo L, Khoury S, Wein A, editors, Incontinence: 4th International Consultation on Incontinence. USA: Health Publications: Plymouth, 2009;1521-1563.

10. Deutekom M, Terra MP, Dobben AC, Dijkgraaf MG, Baeten CG, Stoker J,et al. Impact of faecal incontinence severity on health domains. Colorectal Dis. 2005; 7:263-9.

11. Alimohammadian M, Ahmadi B, Janani L, Mahjubi B. Suffering in silence: a community-based study of fecal incontinence in women. Int J Colorectal Dis. 2014; 29:401-6.

12. Anu E, Sukria N. Endoanal ultrasound assessment of sphincter defects and thinning-correlation with anal manometry. Arab J Gastroenterol. 2014;15:27-31.

13. Terra MP, Deutekom M, Beets-Tan RG, Engel AF, Janssen LW, Boeckxstaens GE, et al. Relationship between external anal sphincter atrophy at endoanal magnetic resonance imaging and clinical, functional, and anatomic characteristics in patients with fecal incontinence. Dis Colon Rectum 2006;49:668-78.

14. Oom DM, West RL, Schouten WR, Steensma AB. Detection of anal sphincter defects in female patients with fecal incontinence: a comparison of 3-dimensional transperineal ultrasound and 2-dimensional endoanal ultrasound. Dis Colon Rectum 2012;55:646-52.

15. Loprinzi PD, Rao SS. Association between fecal incontinence and objectively measured physical activity in u.s. Adults. N Am J Med Sci. 2014;6:575-9.

16. Andrews $\mathrm{CN}$, Bharucha AE. The etiology, assessment, and treatment of fecal incontinence. Nat Clin Pract Gastroenterol Hepatol. 2005;2:516-25.

17. Pretlove SJ, Th ompson PJ, Toozs-Hobson PM, Radley S, Khan KS. Does the mode of delivery predispose women to anal incontinence in the first year postpartum? A comparative systematic review. BJOG 2008;115:421-34.

18. Guzmán Rojas RA, Shek KL, Langer SM, Dietz HP. Prevalence of anal sphincter injury in primiparous women. Ultrasound Obstet Gynecol. 2013; 42:461-6.

19. Borello-France D, Burgio KL, Richter HE, Zyczynski $\mathrm{H}$, Fitzgerald MP, Whitehead W, et al. Fecal and urinary incontinence in primiparous women. Obstet Gynecol. 2006;108:863-72. 
20. Oberwalder M, Connor J, Wexner SD. Meta-analysis to determine the incidence of obstetric anal sphincter damage. Br J Surg. 2003;90:1333-7.

21. Rao SS. Pathophysiology of adult fecal incontinence. Gastroenterology 2004;126(1 Suppl 1):S14-22.

22. Kim YS, Weinstein M, Raizada V, Jiang Y, Bhargava $\mathrm{V}$, Rajasekaran MR, et al. Anatomical disruption and length-tension dysfunction of anal sphincter complex muscles in women with fecal incontinence. Dis Colon Rectum 2013;56:1282-9.

23. Parés D, Iglesias M, Pera M, Pascual M, Torner A, Baró T, et al. Expression of estrogen and progesterone receptors in the anal canal of women according to age and menopause. Dis Colon Rectum 2010;53:1687-91. 